\title{
Digital Fabrication and Assistive Technology
}

\author{
Shahbaz Ahmed ${ }^{1}$, Achraf Ben Jamaa ${ }^{2}$ \\ ${ }^{1}$ Mada Center \\ ${ }^{2}$ Ibtechar
}

Mada Innovation Program aims to encourage innovators to create Arab technological solutions for people with disabilities and the elderly in Qatar and the Arab world. This program seeks to support and develop innovations and skills necessary to manufacture assistive technology devices and to raise awareness in this field among innovators. Mada Center offers its vast experience and, in this field, in addition to creating the appropriate environment to encourage innovation and improving solutions to meet the best international standards.

The MadaFablab ${ }^{8}$ environment and digital fabrication technologies offer people with functional limitations (PFLs) opportunities to develop their skills and enhance their technical and hands-on knowledge, as well as become entrepreneurs. Digital fabrication has major inclusion and well-being benefits for people with disabilities since its supports them to join the workforce. Providing accessible fab labs to PFL will help them develop their own assistive technology tools and contribute to society with new creative ideas.

There are different types of technology in digital fabrication, however, they all serve the same purpose which is creating or modifying a physical object based on computergenerated designs. These technologies are not new, and some of the machines have been widely used in industrial mass manufacturing for many years. Nowadays, these technologies became realistically affordable to the point that hobbyists can get their own equipment.

Main digital fabrication technologies include 3D printers, which can create tangible objects from digital models by building up many layers of melted complex materials. This technology is one of the most known digital fabrication technologies, as it is very user-friendly and commonly used in schools. Laser cutters, on the other side, can cut or etching a wide range of materials in a pattern numerically controlled by software. This technology uses a high-power density resulting in rapid heating, melting, or vaporizing of the materials. This allows a level of precision that few professional persons can achieve with traditional tools. Computer Numerical Control (CNC) routers are the opposite of $3 \mathrm{D}$ printers, as their work is mainly to remove materials rather than build

\footnotetext{
${ }^{8}$ MadaLab https://mip.mada.org.qa/madalab/
} 


\section{Nafath}

Issue 17 - May 2021

them up. This technology uses a computer-controlled cutting tool to subtract away the material to create an object defined on a computer.

The conventional assistive devices and aids used by PFL are often expensive, and in some countries, getting them is difficult and takes a long time. They are also normally mass-produced, meaning they cannot adjust to the customized needs of the user. By the time a device is acquired, it may already be unfit for purpose, leading to high abandonment rates for assistive technologies. This is both frustrating for the user and a waste of resources, but it also means that people with disabilities who might be capable of working with the correct assistive technologies are unable to do so. One of the most promising examples of supporting PFLs in digital fabrication is through DIY Assistive Technologies (DIY-AT). Because digital fabrication allows products to be rapidly customized at little cost, there is huge potential for the customization of existing assistive technologies or even the creation of entirely new assistive devices.

For example, we can use a laser cutter to create a small device to help open bottles, make modifications on a wheelchair, or even prosthetic limbs. If it breaks, or your requirements change, you can easily make another one by creating spare parts using the CNC machine.

A further benefit of digital fabrication is that the digital files used to create designs are often shared freely online. These designs can be downloaded and produced by anybody with the right equipment, anywhere in the world. The designs can also be modified, meaning that it is not necessary to start from scratch since an existing design can be downloaded and modified to meet users' needs.

One example of real-life practices for the DIY Assistive technology centers is MadaFablab (Al Thani et al., 2019) which is the first hands-on inclusive technology and digital fabrication space in the world. The lab contains all the digital fabrication machines required to create prototypes. Moreover, the lab furniture was customized to suit people with functional limitations and fully machined using CNC machines and laser cutters.

\section{References}

Al Thani, D., Al Tamimi, A., Othman, A., Habib, A., Lahiri, A., \& Ahmed, S. (2019, December). Mada Innovation Program: A Go-to-Market ecosystem for Arabic Accessibility Solutions. In 2019 7th International conference on ICT \& Accessibility (ICTA) (pp. 1-3). IEEE. 